\title{
FACTORES ASOCIADOS AL NIVEL DE CONOCIMIENTO Y ACTITUDES SOBRE SIGNOS Y SÍNTOMAS DE ALARMA EN GESTANTES ATENDIDAS POR TELECONSULTA EN EL INSTITUTO NACIONAL MATERNO PERINATAL DURANTE EL AÑO 2020
}

\author{
FACTORS ASSOCIATED WITH THE LEVEL OF KNOWLEDGE AND \\ ATTITUDES ABOUT ALARM SIGNS AND SYMPTOMS IN PREGNANCY \\ CARED FOR BY TELECONSULTATION AT THE NATIONAL MATERNAL \\ PERINATAL INSTITUTE DURING THE YEAR 2020
}

Dayanara Torres-Castillo , Juan Carlos Roque Quezada²

\begin{abstract}
RESUMEN
Objetivo: determinar los factores asociados al nivel de conocimiento y actitudes sobre signos y síntomas de alarma en gestantes atendidas por teleconsulta en el Instituto Nacional Materno Perinatal durante el año 2020. Materiales y métodos: el estudio es de tipo cuantitativo, observacional, analítico, transversal y retrospectivo. Se cuenta con una población de 1000 gestantes y se toma como muestra 214 gestantes atendidas por teleconsulta del Instituto Nacional Materno Perinatal. Se aplicó un instrumento de 28 preguntas de selección múltiple. Resultados: el 88,3\% de las gestantes presenta un nivel de conocimiento alto y un $75,7 \%$ una actitud favorable. Los factores asociados a una actitud favorable fueron: estado civil $(p=0,013)$, paciente añosa $(p=0,002)$, abortos previos $(p<0,001)$, paridad previa $(p=0,001)$ y gestaciones previas $(p<0,001)$. Para nivel de conocimiento, encontramos asociación: estado civil $(p<0,001)$ y paciente añosa $(p<0,001)$. Conclusiones: Los factores asociados significativamente a una actitud favorable y a un alto nivel de conocimiento fueron: estado civil y paciente añosa.
\end{abstract}

Palabras clave: Teleconsulta, atención prenatal, signos y síntomas, alarma, embarazo. (Fuente: DeCS BIREME).

\begin{abstract}
Objective: determine the factors associated with the level of knowledge and attitudes about alarm signs and symptoms in pregnant women treated by remote consultation at the Instituto Nacional Materno Perinatal during the year 2020. Materials and methods: the study is quantitative, observational, analytical, cross-sectional and retrospective. There is a population of 1000 pregnant women and a sample of 214 pregnant women attended by remote consultation from the Instituto Nacional Materno Perinatal. An instrument of 28 multiple-choice questions was applied. Results: $88.3 \%$ of pregnant women presented a high level of knowledge and $75.7 \%$ presented a favorable attitude. Factors associated with a favorable attitude were marital status $(p=0.013)$, elderly patient $(p=0.002)$, previous abortions $(p<0.001)$, previous parity $(p=0.001)$ and previous pregnancies $(p<0.001)$. For the level of knowledge, an association was found marital status $(p<0.001)$ and elderly patient $(p<0.001)$. Conclusions: The factors significantly associated with a favorable attitude and a high level of knowledge: marital status and elderly patient
\end{abstract}

Keywords: Remote consultation, prenatal care, signs and symptoms, alarm, pregnancy. (Source: MeSH NLM).

\section{INTRODUCCIÓN}

Desde los primeros informes de la enfermedad por Coronavirus 2019 (COVID-19) y la identificación del nuevo coronavirus que causa el síndrome respiratorio agudo severo (SARS-CoV-2), a la actualidad la infección se ha extendido a más de 130 millones de casos confirmados en todo el planeta y dejando un saldo de 2 millones de muertes ${ }^{1}$.
Debido a que el virus es altamente contagioso y rápidamente transmisible de persona a persona, los gobiernos de los Estados tomaron diversas acciones para mitigar la propagación, entre ellas el distanciamiento social y la cuarentena, lo cual produjo el cierre de la consulta externa de todos los establecimientos de salud. $Y$ dado que la consulta externa era el principal espacio donde se realizaban los controles prenatales, esta se vio gravemente afectada

Facultad de Medicina Humana, Universidad Ricardo Palma. Lima, Perú. Bachiller Médico-Cirujano.

2 Instituto de Investigación de Ciencias Biomédicas (INICIB). Lima, Perú; Facultad de Medicina Humana. Universidad Ricardo Palma. Médico-Cirujano. Magister en Medicina

iD Código ORCID: https://orcid.org/0000-0002-6251-7043, Dayanara Torres Castillo

Citar como: Torres-Castillo D, Roque Quezada JC. Factores asociados al nivel de conocimiento y actitudes sobre signos y síntomas de alarma en gestantes atendidas por teleconsulta en el Instituto Nacional Materno Perinatal durante el año 2020. Rev Peru Investig Matern Perinat. 2021;10(2): 17-23 DOI https://doi.org/10.33421/inmp.2021232

Recibido: 10-05-2021 Aceptado: 30-06-2021 
llevando a muchas gestantes a no iniciar, o en algunos casos, a no completar sus controles prenatales ${ }^{2,3}$.

La atención prenatal o control prenatal es un espacio donde los profesionales de la salud brindan apoyo, atención e información a las madres sobre cómo se va desarrollando su embarazo ${ }^{4}$.

Las embarazadas representan una población de riesgo durante los brotes de enfermedades infecciosas, ya que los cambios fisiológicos y mecánicos las hacen susceptibles a las infecciones en general, particularmente las del sistema cardiorrespiratorio y fomentan la rápida progresión a insuficiencia respiratoria ${ }^{5}$.

Por ese motivo, la telesalud representa un método para optimizar la atención y mitigar el riesgo de exposición de pacientes en este contexto. Si bien, las teleconsultas no reemplazan totalmente la atención presencial, sí ofrecen un medio para reducir la exposición de pacientes y personal de salud ${ }^{6}$.

El Instituto Nacional Materno Perinatal se basó en la experiencia del seguimiento remoto de Francia $^{7}$ y la individualización de la atención según factores de riesgo de EEUU para incorporar la teleconsulta a un modelo mixto de atención. Para finales de abril de 2020, se aprobó el Proyecto de Teleorientación para pacientes de bajo riesgo y atención presencial restringida para pacientes con alto riesgo de complicación. Durante cinco meses, se han logrado atender 5596 teleconsultas y 638 consultas presenciales.

La comunicación sobre la condición médica y las posibles complicaciones se realizaron mediante WhatsApp, entre las gestantes y los médicos gineco-obstetras del instituto estableciendo mecanismos para brindar una orientación clara y sencilla a toda gestante, reforzando la identificación de signos y síntomas de alarma ${ }^{8}$.

Los signos y síntomas de alarma en el embarazo son: cefalea, tinnitus, visión borrosa, náuseas y vómitos, ausencia de los movimientos fetales, hinchazón de pies, pérdida de sangre por vagina o genitales, fiebre, o la presencia de contracciones uterinas antes de las 37 semanas. Estos son los únicos motivos por los que la paciente debería acudir a la emergencia ${ }^{9}$. Muchos de estos síntomas nos alertan de posibles complicaciones que pueden llevar a la muerte de la madre, del niño, o de ambos.

En Perú, para el año 2020, los trastornos hipertensivos y las hemorragias obstétricas representan las principales causas de muerte materna directa, pero la causa de muerte materna indirecta es el COVID-1910.

El objetivo de esta investigación fue determinar los factores asociados al nivel de conocimiento y actitudes sobre signos y síntomas de alarma en gestantes atendidas por teleconsulta en el Instituto Nacional Materno Perinatal durante el año 2020.

\section{MATERIALES Y MÉTODOS}

Se realizó un estudio de tipo cuantitativo, observacional, analítico, transversal y retrospectivo.

La población estuvo conformada por 1000 gestantes atendidas por teleconsultas del Servicio de Ginecologia y Obstetricia del Instituto Nacional Materno Perinatal durante el año 2020.

Para el cálculo de tamaño muestral, se empleó el paquete estadístico Epidat, dando como resultado 205 , pero la muestra real fue de 214 gestantes.

Se procedió a realizar la recolección de datos por medio de una encuesta adaptada del cuestionario de la tesis de Escaleras Ltitulada "Conocimiento y y actitudes sobre signos y síntomas de alarma en gestantes que acuden al Hospital Universitario de Motupe", LojaEcuador, durante el año 2018.

La encuesta está estructurada de la siguiente manera: datos sociodemográficos (4 ítems), datos obstétricos (4 ítems), signos y síntomas más frecuentes (1 ítem), nivel de conocimiento (16 ítems) y actitudes (12 ítems). Como se mencionó anteriormente, para medir la variable conocimiento, la encuesta se subdividió en 16 preguntas, dando una suma total de 62,08 puntos. La clasificación de las respuestas se agrupó de la siguiente manera: alto (hasta 37,3 puntos), medio (37,4 - 58,6 puntos) y bajo (58,7 a más puntos). Para medir la variable actitud, la encuesta constó de 12 preguntas, cada una de ellas con 05 alternativas estructuradas bajo el sistema Likert, con una valoración de 1 a 5 puntos dependiendo de las declaraciones del encuestado y dando una puntuación total de 60 puntos. Las respuestas se clasificaron en grupos de la siguiente manera: favorable (42 - 60 puntos), indiferente (29 - 41 puntos) y desfavorable (12 - 28 puntos).

Como criterios de inclusión, se tomó en cuenta a todas las gestantes que dieron su consentimiento informado para participar en la investigación y contaron con acceso a internet y a la red social "WhatsApp".

Como criterios de exclusión, no contabilizamos a las gestantes cuyo parto fueron previos a la distribución de la encuesta, a las gestantes con habilidades especiales, a las gestantes analfabetas, a las gestantes con patologías psiquiátricas, a las gestantes cuya única lengua no fue el castellano, y a las gestantes que han contestado de forma incompleta el cuestionario. 
Una vez recolectados los datos, se procedió al análisis de los mismos mediante tablas y gráficos usando el paquete estadístico SPSS Vs 25. Para las variables cualitativas, se reportó la media y desviación estándar.

Para la estadística inferencial, se empleó las pruebas estadísticas Chi cuadrado y Exacta de Fisher, tomados como asociaciones estadísticamente significativas: P-valores inferiores al alfa crítico de 0.05. Empleando un modelo de regresión logística, se realizó el cálculo del RP crudo y sus respectivos intervalos de confianza al $95 \%$ para las variables independientes.

\section{RESULTADOS}

La encuesta se realizó a 214 gestantes. La muestra tiene una edad promedio de 29 años. El 75,2\% respondió conviviente como estado civil y el $46,7 \%$ alcanzó un nivel educativo de secundaria completa. El nivel socioeconómico predominante en la muestra fue el bajo con un $95,3 \%$.
Con respecto a sus antecedentes ginecológicos: las gestantes cuentan con un promedio de 3 gestaciones previas. La mediana de 0 abortos es lo que nos indica que la mayoría no ha presentado abortos previos a lo largo de su vida y su promedio de paridad es de 1, lo que se interpreta que todas las mujeres han parido al menos una vez, por vía vaginal o por cesárea.

Se preguntó cuál es el trimestre de inicio de su control prenatal, el $54,7 \%$ de las encuestadas inició en el segundo trimestre de su embarazo. Esto se debe principalmente a que el servicio de teleconsulta tomó un tiempo en instalarse y ya las gestantes presentaban un embarazo avanzado.

Adicionalmente, se les preguntó si presentaron en el embarazo actual algún signo o síntoma de alarma, y el 53,3\% contestó positivamente, siendo el signo/ síntoma más frecuente "náuseas y vómitos" en un 16,3\% (Tabla 1).

Tabla 1: Datos característicos de la población de gestantes atendidas por teleconsulta en el Instituto Nacional Materno Perinatal (n=214), durante el año 2020.

\begin{tabular}{|c|c|c|}
\hline Edad & \multicolumn{2}{|c|}{29 años RI: 26 - 33} \\
\hline \multirow{4}{*}{ Estado Civil } & Soltera & $5(2,3 \%)$ \\
\hline & Casada & $47(22 \%)$ \\
\hline & Divorciada & $1(0,5 \%)$ \\
\hline & Conviviente & $161(75,2 \%)$ \\
\hline \multirow{5}{*}{ Grado de instrucción } & Primaria completa & $4(1,9 \%)$ \\
\hline & Secundaria completa & $100(46,7 \%)$ \\
\hline & Secundaria incompleta & $14(6,5 \%)$ \\
\hline & Superior universitaria & $31(14,5 \%)$ \\
\hline & Superior técnica & $65(30,4 \%)$ \\
\hline Número de gestaciones & \multicolumn{2}{|c|}{3 gestaciones RI: 2 - 3} \\
\hline Abortos previos & \multicolumn{2}{|c|}{0 abortos RI: 0 - 1} \\
\hline Paridad & \multicolumn{2}{|c|}{1 partos RI: 0 - 2} \\
\hline \multirow{2}{*}{ Nivel socioeconómico } & Nivel Bajo & $204(95,3 \%)$ \\
\hline & Nivel Medio & $10(4,7 \%)$ \\
\hline \multirow{3}{*}{ Inicio de control prenatal } & $1^{\circ}$ trimestre & $87(40,7 \%)$ \\
\hline & $\mathrm{Ii}^{\circ}$ trimestre & $117(54,7 \%)$ \\
\hline & $\mathrm{III}^{\circ}$ trimestre & $10(4,6 \%)$ \\
\hline \multirow{2}{*}{ Presencia de signos de alarma } & $\mathrm{Si}$ & $114(53,3 \%)$ \\
\hline & No & $100(46,7 \%)$ \\
\hline \multirow{3}{*}{ Nivel de conocimiento } & Alto & $189(88,3 \%)$ \\
\hline & Medio & $25(11,7 \%)$ \\
\hline & Bajo & $0(0 \%)$ \\
\hline \multirow{3}{*}{ Actitud } & Desfavorable & $0(0 \%)$ \\
\hline & Indiferente & $52(24,3 \%)$ \\
\hline & Favorable & $162(75,7 \%)$ \\
\hline
\end{tabular}

Fuente: Encuesta aplicada a las gestantes atendidas por teleconsulta en INMP durante el año 2020. 
Tabla 2: Análisis bivariado y multivariado de las variables asociadas a actitud sobre signos y síntomas del embarazo en gestantes atendidas por teleconsulta en el Instituto Nacional Materno Perinatal durante el año 2020.

\begin{tabular}{|c|c|c|c|c|}
\hline & \multicolumn{2}{|c|}{ Actitud } & \multirow{2}{*}{ RP crudo (IC 95\%) } & \multirow{2}{*}{ RP ajustado (IC 95\%) } \\
\hline & Indiferente & Favorable & & \\
\hline \multicolumn{5}{|l|}{ Nivel Educativo } \\
\hline Educación superior & $22(22,9 \%)$ & $74(77,1 \%)$ & 1 & 1 \\
\hline Educación básica & $30(25,4 \%)$ & $88(74,6 \%)$ & $0,991(0,950-1,033)$ * & $0,983(0,950-1,017)$ * \\
\hline \multicolumn{5}{|c|}{ Nivel Socioeconómico } \\
\hline Nivel Medio & $3(30 \%)$ & $7(70 \%)$ & 1 & 1 \\
\hline Nivel Bajo & $49(24 \%)$ & $155(76 \%)$ & $1,022(0,918-1,138)$ * & $1,023(0,949-1,102)$ \\
\hline \multicolumn{5}{|l|}{ Estado civil } \\
\hline No casada & $46(27,5 \%)$ & $121(72,5 \%)$ & $0,949(0,910-0,989)$ ** & $0,968(0,933-1,003)$ * \\
\hline Casada & $6(12,8 \%)$ & $41(87.2 \%)$ & 1 & 1 \\
\hline \multicolumn{5}{|c|}{ Número de gestaciones } \\
\hline Más de 1 gestación & $26(14,1 \%)$ & $115(71,4 \%)$ & 1 & 1 \\
\hline Primera gestación & $26(86,7 \%)$ & $4(13,3 \%)$ & $0,746(0,703-0,792)^{* *}$ & $0,738(0,690-0,789)$ ** \\
\hline \multicolumn{5}{|l|}{ Paciente añosa } \\
\hline Añosa & $2(7,7 \%)$ & $24(92,3 \%)$ & 1 & 1 \\
\hline No añosa & $50(26,6 \%)$ & $138(73,4 \%)$ & $0,935(0,897-0,975)$ ** & $0,955(0,917-0,995)$ ** \\
\hline \multicolumn{5}{|l|}{ Paridad previa } \\
\hline $\mathrm{Si}$ & $27(17,6 \%)$ & $126(82,4 \%)$ & 1 & 1 \\
\hline No & $25(41 \%)$ & $36(59 \%)$ & $0,917(0,871-0,967)$ ** & $0,965(0,928-1,003)^{*}$ \\
\hline \multicolumn{5}{|l|}{ Abortos previos } \\
\hline $\mathrm{Si}$ & $6(7,7 \%)$ & $72(92,3 \%)$ & 1 & 1 \\
\hline No & $46(33,8 \%)$ & $90(66,2 \%)$ & $0,911(0,878-0,944)$ ** & $1,026(0,985-1,069)^{*}$ \\
\hline
\end{tabular}

*p-valor $>0,05$ : variables no presentan asociación estadísticamente significativa

${ }^{* *} p$-valor $<0,05$ : variables presentan asocación estadísticamente significativa. ${ }^{1}$ Referencia.

Como el objetivo principal fue determinar qué factores se asociaban al nivel de conocimiento y actitudes sobre signos y síntomas del embarazo, se examinó las dos variables de forma individual.

Por análisis bivariado, encontramos que los factores estadísticamente significativos para una actitud favorable fueron: estado civil, número de gestaciones, paciente añosa, abortos previos y paridad previa, y los factores estadísticamente significativos para un nivel de conocimiento alto: estado civil y paciente añosa.

Lo interpretamos como que estar casada genera una mejor actitud sobre los signos y síntomas de alarmas con respecto a aquellas gestantes que no lo están (RP crudo 0,95, IC95\% 0,91-1,98, p=0,013). Haber estado embarazada más de una vez mejora la actitud sobre los signos y síntomas de alarmas, con respecto a quienes ésta es su primera gestación (RP ajustado 0,74, IC95\% 0,69-0,78, p<0,001). El haber tenido un aborto previo, predispone a una mejor actitud con respecto a aquellas gestantes sin abortos previos (RP crudo 0,91, IC95\% 0,87-0,94, $p<0,001)$. Por último, una gestante con al menos un parto tiende a presentar una mejor actitud con respecto a una que todavía no ha dado a luz (RP crudo 0,09, IC95\% 0,87-0,97, $\mathrm{p}<0,001$ ) (Tabla 2).

Con respecto a la variable nivel de conocimiento, se encontró también por análisis bivariado, que estar casada predispone a tener un mejor nivel de conocimiento con respecto a aquellas que son solteras o convivientes (RP ajustado 1,15, IC95\% 1,08-1,22, $p<0,001)$. Y que una edad de 35 años, predispone a tener un mejor nivel de conocimiento (RP ajustado 1,11, IC95\% 1,06-1,17) (Tabla 3).

\section{DISCUSIÓN}

Comparando resultados, encontramos que las gestantes presentaron en su mayoría un nivel de conocimiento alto en un $88,3 \%$ (189 casos). El $11,7 \%$ (25 casos) presentó un nivel de conocimiento medio y ninguna gestante presentó un nivel de conocimiento bajo. Estos resultados son diferentes a los encontrados en la tesis de Escaleras $\mathrm{L}^{11}$. Ella presenta una prevalencia de $68,84 \%$, pero en gestantes con un nivel de conocimiento medio y en segundo lugar se encontraban las gestantes con un nivel alto que correspondía a un $31,16 \%$. El estudio 
Tabla 3: Análisis bivariado y multivariado de las variables asociadas al nivel de conocimiento sobre signos y síntomas del embarazo en gestantes atendidas por teleconsulta en el Instituto Nacional Materno Perinatal durante el año 2020

\begin{tabular}{|c|c|c|c|c|}
\hline & \multicolumn{2}{|c|}{ Actitud } & \multirow{2}{*}{ RP crudo (IC 95\%) } & \multirow{2}{*}{ RP ajustado (IC 95\%) } \\
\hline & Medio & Alto & & \\
\hline \multicolumn{5}{|l|}{ Nivel Educativo } \\
\hline Educación superior & $8(8,3 \%)$ & $88(91,7 \%)$ & 1 & 1 \\
\hline Educación básica & $17(14,4 \%)$ & $101(85,6 \%)$ & $1,056(0,979-1,139)$ * & $1,039(0,967-1,117)$ * \\
\hline \multicolumn{5}{|c|}{ Nivel Socioeconómico } \\
\hline Nivel Medio & $2(20 \%)$ & $8(80 \%)$ & 1 & 1 \\
\hline Nivel Bajo & $23(11,3 \%)$ & $181(88,7 \%)$ & $0,927(0,751-1,144)$ * & $0,842(0,691-1,026)$ * \\
\hline \multicolumn{5}{|l|}{ Estado civil } \\
\hline No casada & $25(15 \%)$ & $142(85 \%)$ & $1,050(1,97-1,205) * *$ & $1,151(1,085-1,221) * *$ \\
\hline Casada & $0(0 \%)$ & $47(100 \%)$ & 1 & 1 \\
\hline \multicolumn{5}{|l|}{ Gestaciones previas } \\
\hline Más de 1 gestación & $21(11,4 \%)$ & $163(88,6 \%)$ & 1 & 1 \\
\hline Primera gestación & $4(13,3 \%)$ & $26(86,7 \%)$ & $1,017(0,907-1,141)^{*}$ & $1,038(0,884-1,220)$ * \\
\hline \multicolumn{5}{|l|}{ Paciente añosa } \\
\hline Añosa & $0(0 \%)$ & $26(100 \%)$ & 1 & 1 \\
\hline No añosa & $25(13,3 \%)$ & $163(86,7 \%)$ & $1,133(1,085-1,183)$ ** & $1,114(1,060-1,170)$ ** \\
\hline \multicolumn{5}{|l|}{ Paridad previa } \\
\hline $\mathrm{Si}$ & $17(11,1 \%)$ & $136(88,9 \%)$ & 1 & 1 \\
\hline No & $8(13,1 \%)$ & $53(86,9 \%)$ & $1,018(0,933-1,111)$ * & $0,974(0,858-1,105)$ * \\
\hline \multicolumn{5}{|l|}{ Abortos previos } \\
\hline $\mathrm{Si}$ & $11(14,1 \%)$ & $67(85,9 \%)$ & 1 & 1 \\
\hline No & $14(10,3 \%)$ & $122(89,7 \%)$ & $0,967(0,891-1,049)$ * & $0,954(0,866-1,051)$ * \\
\hline
\end{tabular}

${ }^{*} p$-valor $>0,05$ : variables no presentan asociación estadísticamente significativa

${ }^{* *}$ p-valor $<0,05$ : variables presentan asocación estadísticamente significativa. ${ }^{1}$ Referencia.

que nos mostró una prevalencia similar fue el de Sulca-Díaz RO, et al ${ }^{12}$. Ellos dieron como resultado que el nivel de conocimiento alto representaba el $70,7 \%$, valor similar al encontrado en nuestro estudio. La diferencia se puede deber a que el estudio de Escaleras $L^{11}$. se realizó en un centro de salud con una población menor a la encuestada en el presente estudio. Por el contrario, nosotros trabajamos con una población mayor y en un centro de salud de mayor complejidad que sigue y monitoriza a sus pacientes constantemente. Características que se repiten en el estudio de Sulca-Díaz RO et al ${ }^{12}$, quienes realizaron su encuesta en un hospital de Nivel III.

Con respecto a la actitud, nuestro trabajo determinó que el $75,7 \%$ (162 casos) de gestantes tenía una actitud favorable frente a los signos y síntomas del embarazo. Valores similares a los encontrados en los trabajos de Escaleras $L^{11}$ y Sulca-Díaz $R O$ et al ${ }^{12}$ con valores de $78,70 \%$ y $95,3 \%$, respectivamente. La similitud en valores sobre una actitud favorable frente a los signos y síntomas de alarma, nos podría indicar que no solo un adecuado conocimiento es suficiente para tener una actitud favorable sobre signos y síntomas de alarma, sino que debemos tomar en cuenta otras variables que no se pueden medir, como, por ejemplo, el "instinto materno" que a pesar de no contar con los adecuados conocimientos sobre los signos o síntomas, una madre sabe cuándo una situación se complica y actúa de forma adecuada en el momento indicado.

Se observa la existencia de una correlación estadísticamente significativa entre el nivel de conocimiento y la actitud frente a los signos y síntomas de alarma en el embarazo ( $p$-valor 0,000): se deduce que a un alto nivel de conocimiento, hay una mejor actitud. Este resultado se asemeja a los encontrados en Tituaña-Flores $E^{13}$ y Cochachin-Minaya A, MejiaMendoza $\mathrm{P}^{14}$. Esta asociación, en realidad, es un resultado que se repite en varias tesis. Lógicamente es de esperarse que al saber más de un tema, las actitudes van a ser las más adecuadas.

Es importante destacar que el 53,3\% (114 casos) de pacientes presentó al menos un signo o síntoma de alarma en el presente embarazo, siendo las "náuseas y vómitos" el signo más frecuente en un 16,4\% (35 casos). Estos resultados son similares a los encontrados en Ticona-Rebagliati $D$ et al $^{9}$. Se encontró un $33 \%$ de gestantes que presentó "náuseas y vómitos intensos 
y repetidos" como el signo de alarma más frecuente durante su embarazo actual. Las "náuseas y vómitos" son el signo/síntoma más frecuente, debido a que existe la teoría de que el aumento de la gonadotropina coriónica humana y los estrógenos están asociados directamente a la aparición de hiperemesis gravídica.

Cabe mencionar que existen dos variables dependientes, pero la variable actitud es la que presenta mayor asociación estadísticamente significativa con mayor cantidad de variables.

Para comprobar las hipótesis planteadas, primero evaluamos la variable actitud que presenta por análisis bivariado una asociación estadísticamente significativa $(p<0,05)$ con las variables: estado civil (RP 0,95, IC95\% $0,92-0,99, p=0,013$ ), paciente añosa (RP 0,93, IC95\% $0,89-0,97, p=0,002$ ), abortos previos (RP 0,91, IC95\% $0,87-0,94, p<0,001$ ), paridad previa (RP 0,91, IC $95 \% 0,87-$ $0,96, p<0,001)$ y gestaciones previas (RP 0,74, IC95\% $0,70-0.79, p<0,001)$, para lo cual se empleó el análisis de regresión de Poisson. Con los valores mencionados, las hipótesis alternas de paridad previa, grupo etario y abortos previos se aceptan y por consiguiente se rechazan las hipótesis nulas de las mismas hipótesis. En cambio, en el análisis multivariado, solo las variables: paciente añosa (RP 0,95, IC95\% 0,91-0,99, p=0,027) y gestaciones previas (RP 0,73, IC95\% 0,69-0,78, $\mathrm{p}<0,001)$ fueron estadísticamente significativas. En diversos estudios realizados en África ${ }^{15-17}$, se aprecia similar asociación, pues las mujeres con embarazos previos, siendo estas multigestas o multíparas, son siempre quienes tienen un mayor conocimiento $\mathrm{y}$, por ende, una mejor actitud. Esto puede deberse a que han experimentado alguna complicación previa que convierte esa experiencia en conocimiento.

Las variables nivel educativo y nivel socioeconómico no son estadísticamente significativas para nuestro estudio. Según el estudio de Pembe $A$ et $a^{18}$, ellos también determinaron que el estado civil no tiene asociación, resultado que difiere de nuestro estudio; pero el nivel educativo sí difiere, pues ellos refieren que a mayor nivel escolar mayor conocimiento y por ende una mejor actitud sobre signos y síntomas de alarma. Ambas variables se consideran estadísticamente significativas en los estudios de Nebiyu Maseresha et al $^{19}$ y Tamang ST et $a l^{20}$, lo cual difiere parcialmente a los datos encontrados en nuestro estudio.

Posteriormente, se realizó el mismo análisis con la variable nivel de conocimiento, encontrándose una asociación estadísticamente significativa con las variables estado civil (RP 0,15, IC95\% 1,09-1,20, $p<0,001$ ) y paciente añosa (RP 0,96, IC95\% 0,89-1,04, p<0,001). Similares resultados vemos en Amenu et $\mathrm{al}^{15} \mathrm{y}$ Tamang ST et al ${ }^{20}$ donde la variable presentar pareja se convierte en una asociación favorable para un alto nivel de conocimiento.
Dando un poco de trasfondo, Perú como muchos países latinoamericanos, es un país considerado en desarrollo, pero principalmente es un país que entra en la categoría de pobre. Para el INEI, durante el año 2020 , el $32,5 \%$ de las madres son convivientes, el $18,5 \%$ son separadas, el $10 \%$ son viudas y el $5,3 \%$ son solteras; y un $26 \%$ han alcanzado una educación superior ${ }^{21}$. Estas cifras aisladas no nos indican mucho, pero si las analizamos en conjunto, observamos que el estado civil, principalmente las madres que no tiene pareja, se asocia a un incremento en el riesgo de malas prácticas antenatales. Por otro lado, si las madres tienen menor nivel educativo hay más riesgo de tener más hijos y si a eso le añadimos un bajo nivel socioeconómico, se traduce en prácticas sexuales tempranas y reproductivas avaladas por su mismo contexto, contribuyendo a la perpetuidad de la pobreza. Si la madre venía de una familia de pocos recursos, al tener un niño, las oportunidades decrecen; porque al haber escasez, hay poca motivación y poco apoyo familiar o abandono del padre del niño. Las adolescentes o jóvenes prefieren continuar con el embarazo al no haber otras alternativas de progresar. En realidad, las variables representan un trasfondo importante que estudiar y que debería seguir estudiándose a mayor escala.

La principal limitación es el sesgo de memoria. Cuando uno desconoce un tema, con frecuencia la memoria manufactura nuevos recuerdos para llenar los espacios vacíos y esto genera un inadecuado llenado de la encuesta, por lo que los datos se vuelven poco confiables.

Otra limitación que se presentó es de localización, al ser unicéntrico, los resultados solo representan una muestra del Instituto. Si bien, es un centro de referencia nacional, no representa toda la población de Lima o de Perú.

Declaración de conflictos de intereses: Los autores declaran no tener conflicto de intereses.

Financiamiento: Autofinanciado.

\section{REFERENCIAS BIBLIOGRÁFICAS}

1. COVID-19 Map [Internet]. Johns Hopkins Coronavirus Resource Center. [cited 2021 Apr 2]. Available from: https:// coronavirus.jhu.edu/map.html

2. CDC. La comunidad, el trabajo y la escuela [Internet]. Centers for Disease Control and Prevention. 2020 [cited 2021 Apr 2]. Available from: https://espanol.cdc.gov/coronavirus/2019-ncov/community/correction-detention/guidance-correctional-detention.html

3. Gonzales-Castillo JR, Varona-Castillo L, Dominguez-Morante MG, Ocaña-Gutierrez VR. Pandemia de la COVID-19 y las Políticas de Salud Pública en el Perú: marzo-mayo 2020. Rev salud pública. 2020 Mar 1;22(2):1-9. 
4. La OMS señala que las embarazadas deben poder tener acceso a una atención adecuada en el momento adecuado [Internet]. [cited 2021 Apr 2]. Available from: https://www. who.int/es/news/item/07-11-2016-pregnant-women-mustbe-able-to-access-the-right-care-at-the-right-time-says-who

5. Dashraath P, Wong JLJ, Lim MXK, Lim LM, Li S, Biswas A, et al. Coronavirus disease 2019 (COVID-19) pandemic and pregnancy. American Journal of Obstetrics \& Gynecology. 2020 Jun 1;222(6):521-31.

6. Aziz A, Zork N, Aubey JJ, Baptiste CD, D'Alton ME, Emeruwa UN, et al. Telehealth for High-Risk Pregnancies in the Setting of the COVID-19 Pandemic. Am J Perinatol. 2020 Jun;37(8):800-8.

7. Vivanti AJ, Deruelle P, Picone O, Guillaume S, Roze J-C, Mulin B, et al. Follow-up for pregnant women during the COVID-19 pandemic: French national authority for health recommendations. J Gynecol Obstet Hum Reprod. 2020 Sep;49(7):101804.

8. Guevara Ríos, E. La atención prenatal en tiempos de COVID-19. Revista Peruana De Investigación Materno Perinatal [Internet]. 2020 [cited 2021 Apr 2];9(3). Available from: https://investigacionmaternoperinatal.inmp.gob.pe/index. php/rpinmp/article/view/208

9. Ticona-Rebagliati D, Torres-Bravo L, Veramendi L, Zafra-Tanaka JH. Conocimientos y prácticas sobre signos de alarma de las gestantes atendidas en el Hospital Nacional Docente Madre- Niño San Bartolomé, Lima, Perú. :8.

10. MINSA. Boletín Epidemiológico del Perú 2020 [Internet]. Ministerio de Salud del Perú; 2020. Available from: https:// www.dge.gob.pe/epipublic/uploads/boletin/boletin_202053. pdf

11. Escaleras Cabrera, Luz Benigna E. Conocimientos y actitudes sobre signos y síntomas de alarma en gestantes que acuden al hospital universitario de motupe. [tesis obtención del título de licenciada en enfermería]. [Loja - Ecuador]: Universidad Nacional de Loja; 2018.

12. Sulca-Diaz R, Peñafiel-Palomino RP, Rosa-Ventura JAD et al. Conocimiento y actitudes de las gestantes sobre los principales signos y síntomas de alarma en el embarazo, Hospital Regional de Ica, enero 2017. Revista médica panacea [internet]. 2017 [cited 2020 Sep 28];6(1). Available from: https://revistas.unica.edu.pe/index.php/panacea/article/view/180

13. Flores ELT, Jácome GEM. Conocimientos y actitudes sobre signos y síntomas de alarma en gestantes atendidas en el centro de salud anidados Otavalo, 2018-2019.

14. Cochachin AG, Mejía PH, Arotoma MI. Conocimientos y actitudes sobre señales de alarma durante el embarazo Hospital Carlos Lanfranco La Hoz - Lima 2016 [Internet] [Tesis para optar por el título profesiol de licenciado en obstetricia]. [Huaraz - Perú]: Universidad Nacional "Santiago Antúnez De Mayolo"; 2017. Available from: http://repositorio.unasam.edu.pe/bitstream/handle/UNASAM/1919/ T033_45731999_T.pdf?sequence=4\&isAllowed=y

15. Amenu G, Mulaw Z, Seyoum T, Bayu H. Knowledge about Danger Signs of Obstetric Complications and Associated Factors among Postnatal Mothers of Mechekel District Health Centers, East Gojjam Zone, Northwest Ethiopia, 2014. Scientifica (Cairo) [Internet]. 2016 [cited 2021 May 19];2016. Available from: https://www.ncbi.nlm.nih.gov/pmc/ articles/PMC4916279/

16. Bolanko A, Namo H, Minsamo K, Addisu N, Gebre M. Knowledge of obstetric danger signs and associated factors among pregnant women in Wolaita Sodo town, South Ethiopia: A community-based cross-sectional study. SAGE
Open Med [Internet]. 2021 Mar 12 [cited 2021 May 19];9. Available from: https://www.ncbi.nlm.nih.gov/pmc/articles/ PMC7958171/

17. Woldeamanuel GG, Lemma G, Zegeye B. Knowledge of obstetric danger signs and its associated factors among pregnant women in Angolela Tera District, Northern Ethiopia. BMC Res Notes [Internet]. 2019 Sep 23 [cited 2021 May 19];12. Available from: https://www.ncbi.nlm.nih.gov/ pmc/articles/PMC6755683/

18. Pembe AB, Urassa DP, Carlstedt A, Lindmark G, Nyström L, Darj E. Rural Tanzanian women's awareness of danger signs of obstetric complications. BMC Pregnancy Childbirth. 2009 Mar 26;9:12.

19. Maseresha N, Woldemichael K, Dube L. Knowledge of obstetric danger signs and associated factors among pregnant women in Erer district, Somali region, Ethiopia. BMC Womens Health [Internet]. 2016 Jun 6 [cited 2021 May 19];16. Available from: https://www.ncbi.nlm.nih.gov/pmc/articles/ PMC4893837/

20. Tamang ST, Dorji T, Yoezer S, Phuntsho T, Dorji P. Knowledge and understanding of obstetric danger signs among pregnant women attending the antenatal clinic at the National Referral Hospital in Thimphu, Bhutan: a cross-sectional study. BMC Pregnancy Childbirth [Internet]. 2021 Feb 2 [cited 2021 May 19];21. Available from: https://www.ncbi.nlm. nih.gov/pmc/articles/PMC7852084/

21. En el Perú hay 8 millones 664 mil madres [Internet]. [cited 2021 May 19]. Available from: https://www.inei.gob. pe/prensa/noticias/en-el-peru-hay-8-millones-664-mil-madres-12868/

\section{Correspondencia:}

Dayanara Torres Castillo

Dirección: Av. Alberto Alexander 2343 -departamento $\mathrm{K}$-, Lince

Correo: dapatoca3011@aol.com

Teléfono: 997450225 